\title{
Medical journals, impact and social media: an ecological study of the Twittersphere
}

\author{
Theodore D. Cosco PhD
}

\section{- Abstract \\ Background: Twitter is an increasingly popular means of research dissemination. I sought to examine the relation between scientific merit and mainstream popularity of general medical journals.}

Methods: I extracted impact factors and citations for 2014 for all general medical journals listed in the Thomson Reuters InCites Journal Citation Reports. I collected Twitter statistics (number of followers, number following, number of tweets) between July 25 and 27, 2015 from the Twitter profiles of journals that had Twitter accounts. I calculated the ratio of observed to expected Twitter followers according to citations via the Kardashian Index. I created the (Fifty Shades of) Grey Scale to calculate the analogous ratio according to impact factor.

Results: Only $28 \%(43 / 153)$ of journals had Twitter profiles. The scientific and social media impact of journals were correlated: in adjusted models, Twitter followers increased by $0.78 \%$ (95\% confidence interval [CI] $0.38 \%-1.18 \%$ ) for every $1 \%$ increase in impact factor and by $0.62 \%(95 \% \mathrm{Cl} 0.34 \%-0.90 \%)$ for every $1 \%$ increase in citations. Kardashian Index scores above the $99 \% \mathrm{Cl}$ were obsverved in $16 \%$ (7/43) of journals, including 6 of the 7 highest ranked journals by impact factor, whereas 58\% (25/43) had scores below this interval. For the Grey Scale, $12 \%$ (5/43) of journals had scores above and 35\% (15/43) had scores below the $99 \% \mathrm{Cl}$.

Interpretation: The size of a general medical journal's Twitter following is strongly linked to its impact factor and citations, suggesting that higher quality research received more mainstream attention. Many journals have not capitalized on this dissemination method, although others have used it to their advantage.
$\mathrm{S}$ ocial media has reached near ubiquity; medical research, researchers and journals are no exception to its pervasiveness. ${ }^{1-7}$ One of the most popular social media platforms is Twitter, with an estimated 302 million monthly active users sending 500 million tweets per day. ${ }^{8}$ Twitter differs from other social media platforms in that posts are limited to 140 or fewer characters. With an emphasis on brevity, Twitter provides a unique opportunity for medical knowledge to be disseminated to the general public. ${ }^{9,10}$ However, people with questionable medical research pedigrees are the stars of Twitter.

With more than 65 million followers, Canadian@justinbieber is one of the most popular Twitter celebrities, narrowly edging out the most popular physician (@bengoldacre) by a margin of 64.5 million followers. Despite widespread distaste among non-Beliebers, it can be argued that The Biebs does display a modicum of musical talent (assuming multiplatinum albums and chart-topping singles ${ }^{11}$ are a proxy for talent). Conversely, the woefully popular @ KimKardashian, who trumps top scientists by more than 33 million followers, falls into the "famous for being famous" trope, alongside fellow Glitterati Paris Hilton and Nicole Richie. The notion that people with dubious levels of talent and questionable means of attaining celebrity can become immensely popular is worrisome. This notion has sparked debate within the scientific community. Do these self-perpetuated self-promoters exist in academia? Are any scientists "renowned for being renowned"? ${ }^{12}$

There has been increasing use of alternative means of quantifying journals' impact, notably using the Altmetric statistic, which conglomerates an article's social media presence through blogs, news outlets, Facebook and Twitter. In response
Competing interests: None declared.

This article has been peer reviewed.

Accepted: Oct. 17, 2015

Print: Dec. 8, 2015

Correspondence to:

Theodore Cosco,

tdcosco@cantab.net

Twitter: @ tdcosco

CMAJ 2015. DOI:10.1503/ cmaj.150976 
to the meteoric unmeritocratic rise of social media celebrities via Twitter, @neilhall_uk developed the playfully dubbed Kardashian Index (K-index) to address these issues in an academic context. ${ }^{12}$ The K-index quantifies the discrepancy between mainstream popularity and scientific merit by examining one's social media profile in relation to one's citations in peer reviewed works.

Continuing in this vein, I propose the (Fifty Shades of) Grey Scale for use with medical journals (in reference to the book, which has sold more than 125 million copies to date, despite being critically lambasted). ${ }^{13}$ Using a similar equation to the K-index, the Grey Scale calculates the ratio of the number of actual to expected followers using journal impact factor (rather than citations, as in the K-index) as the predictor variable. Journal impact factor and total citations are closely related. Impact factor is the ratio of total citations to the number of articles published by the journal, which adjusts for journals that have many more, or fewer, citable publications (e.g., weekly or bimonthly journals). ${ }^{14}$

Unpacking the mechanisms of Twitter celebrity is difficult. Personal Twitter profiles often include humour, wit and other attributes not normally attributed to the reporting of a new paper, as per general medical journal Tweets. By eliminating the individuality of the Tweet, looking only at medical journals' Twitter profiles rather than individual researchers', a more direct examination of the relation between Twitter celebrity and scientific merit is possible. Although Tweets linking to papers have been associated with greater citations than non-Tweeted papers, ${ }^{15}$ whether or not this translates into greater Twitter followings for the authors and the journal in which the paper was published has yet to explored. The relation between the number of Twitter followers and impact factor scores has recently been investigated in urology journals, where nonsignificant correlations between the number of Twitter followers and the impact factor of the journal were found. ${ }^{16}$

The current study seeks to examine whether scientific merit (captured by journal impact factor and citations) translates into Twitter celebrity (i.e., number of followers) in general medical journals.

\section{Methods}

\section{Data source}

The Thomson Reuters InCites Journal Citations Report (https://jcr.incites.thomsonreuters.com) is a platform used to compare statistics on peerreviewed journals, notably impact factor and citations. Journals in the "Medical, General \&
Internal" Web of Science category schema were selected and their 2014 data extracted.

Twitter profiles for all of the journals identified in the Journal Citations Report were searched between July 25 and 27, 2015, and data extracted on number of followers, number following and tweets sent.

\section{Statistical analysis}

All procedures were carried out in Stata 13. Given the nonnormal nature of the data, nonparametric and log-transformed procedures were used throughout.

Spearman correlations were conducted to examine the relation of number of followers with journal impact factor and citations.

Log-log regression models were conducted to examine the relation of number of followers with journal citations and impact factor in unadjusted and adjusted (for number of tweets) models. More active Twitter accounts are generally associated with greater numbers of followers; therefore, regression models were adjusted for number of Tweets.

Kardashian Index scores ${ }^{12}$ were calculated for each journal using Hall's equation

$$
K-\text { Index }=F(\mathrm{a}) / 43.3 C^{0.32}
$$

where $F(a)$ is the actual number of Twitter followers and $C$ is the number of citations.

(Fifty Shades of) Grey Scale scores were calculated using a log-adjusted regression equation for estimating the number of expected followers, derived from the dataset in the present study (i.e., the data for Twitter followers, tweets and impact factors that were collected on the general medical journals were used to find the best-fitting regression equation, which yielded the coefficients 0.79 for the observed association of tweets and 0.78 for the observed association of impact factor with followers) as follows:

$$
F(\mathrm{e})=T^{0.79}+I^{0.78}
$$

where $F(\mathrm{e})$ is the expected number of Twitter followers, $T$ is the number of tweets and $I$ is the impact factor of the journal.

Thus, the Grey Scale is a measurement of the degree to which any given data point diverges from the observed average relation of tweets and impact factor with followers, analogous to the Kardashian Index:

$$
\text { Grey Scale }=F(\mathrm{a}) / T^{0.79}+I^{0.78}
$$

As per Hall, ${ }^{12} \mathrm{~K}$-index scores of more than 5 suggest a "Science Kardashian"; that is, a dispro- 
portionately high number of followers when compared with citations. In addition to Hall's threshold, journals that fell beyond $99 \%$ confidence intervals (CIs) were identified in the K-index and Grey Scale.

\section{Results}

The Thomson Reuters InCites Journal Citations Reports identified 153 journals in their general and internal medical categories, of which 43 had Twitter accounts. Twitter accounts for publishers of the journals were not included (Appendix 1, available at www.cmaj.ca/lookup/suppl/doi: 10.1503/cmaj.150976/-/DC1).

Journal characteristics and Twitter characteristics varied greatly among the 43 journals with Twitter accounts. Impact factors ranged from 0.24 to 55.87, with a mean of 6.56 (SD 11.82) and median of 2.26. Total journal citations for 2014 ranged from 1269 to 268 652, with a mean of 26291 (SD 58 074) and median of 4327. Journals' total number of Twitter followers ranged from 4 to 277451 , with a mean of 24065 (SD 59805 ) and a median of 1427 . Journals' total number of profiles followed on Twitter ranged from 0 to 5087 with a mean of 682 (SD 979) and a median of 289. Journals' total number of tweets ranged from 3 to 20821 with a mean of 3027 (SD 4378) and a median of 994.

Significant positive Spearman correlations were seen between number of followers and journal impact factor $(r=0.68, p<0.001)$ and journal citations $(r=0.69, p<0.001)$.

Unadjusted log-log regression models showed significant relations between number of followers and journal impact factor $(p<0.001$; Figure 1$)$ and journal citations $(p<0.001$; Figure 2$)$. A $1.00 \%$ increase in impact factor was associated with a $1.46 \%(95 \%$ CI $1.00 \%-1.93 \%)$ increase in Twitter followers. A $1.00 \%$ increase in journal citations was associated with a $1.09 \%(95 \%$ CI $0.75 \%-1.44 \%$ ) increase in Twitter followers. In $\log -\log$ regression models adjusted for number of tweets, a $1.00 \%$ increase in impact factors was associated with a $0.78 \%$ (95\% CI $0.38-1.18)$ increase in Twitter followers, and a $1.00 \%$ increase in journal citations was associated with a $0.62 \%$ (95\% CI $0.34 \%-0.90 \%$ ) increase in Twitter followers. The variance in number of followers explained was roughly equal in the adjusted citations $\left(R^{2}=0.76, p<0.001\right)$ and impact factor models $\left(R^{2}=0.75, p<0.001\right)$.

The average K-index score was 18.23 (SD 38.14). Fifteen journals fit the criteria for Hall's $s^{12}$ Science Kardashians (i.e., K-index $\geq 5$ ) (Figure 3). Using the $99 \%$ CI method (i.e., K-index $\geq 33.22$ ), 7 journals were Science Kardashians.
Twenty-five journals fell below the $99 \%$ CI (i.e., K-index $\leq 3.25$ ).

The average Grey Scale score was 25.62 (SD 52.64). Five journals fell beyond the upper $99 \%$ CI (i.e., Grey Scale score $\geq 46.30$ ) and 15 below (i.e., Grey Scale score $\leq 4.94$ ). More highly ranked journals were more likely to have high $\mathrm{K}$-index (Figure 3) and Grey Scale (Figure 4) scores.

\section{Interpretation}

A strong and independent relation was shown between general medical journals' number of Twitter followers, the journal's impact factor and total number of citations.

Owing to the ecological nature of the data, establishing a causative relation is not possible. Furthermore, the journal citation and impact factor data were collected for 2014, whereas the Twitter data were collected in July 2015. The directionality of the influence of impact factor and followers cannot be established conclusively because of these limitations.

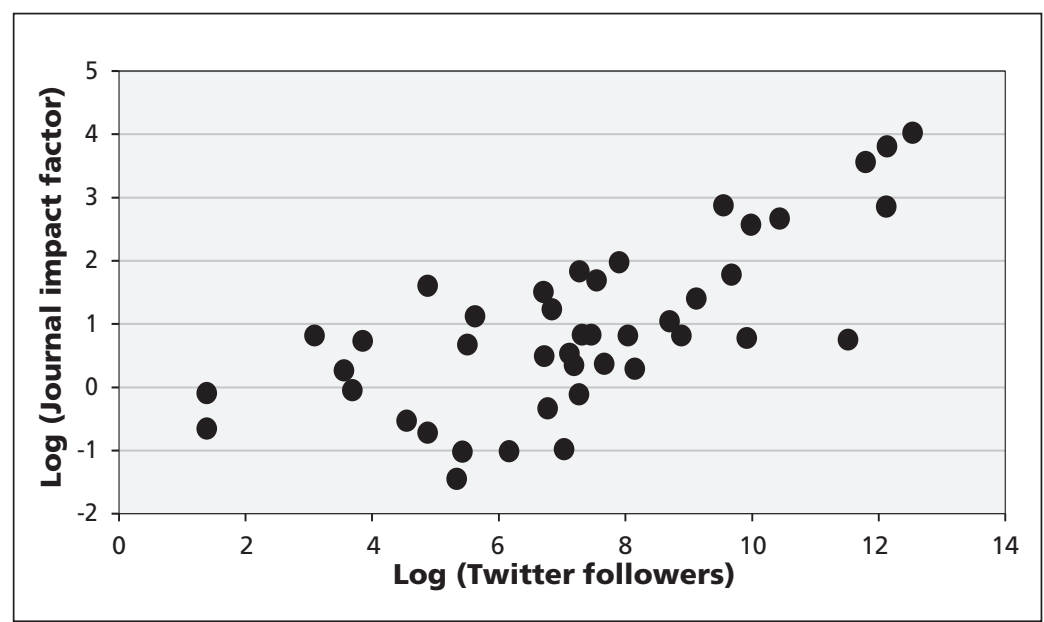

Figure 1: Log-log regression scatterplot of Twitter followers and journal impact factor.

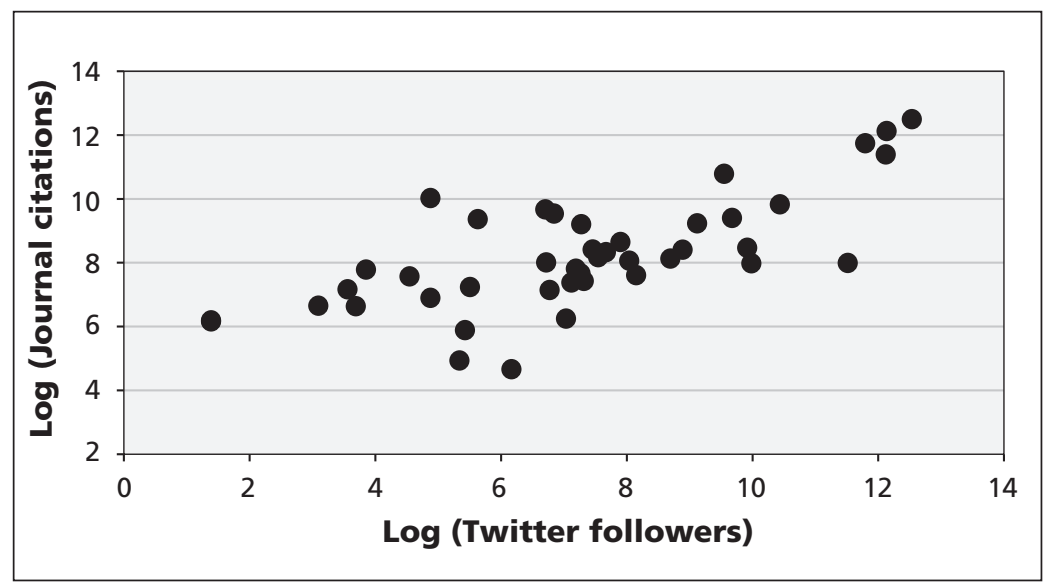

Figure 2: Log-log regression scatterplot of Twitter followers and journal citations. 
Only $28.1 \%$ (43/153) of journals indexed by the Thomson Reuters InCites Journal Citations Report in the "Medical, General \& Internal" Web of Science category had Twitter profiles. Several journals had links to their publisher's Twitter profile, such as all of the BioMed Central journals, but these were excluded from the current study; therefore, some journals with social media profiles were excluded from these analyses.

The application of the K-index to journals, rather than to individuals, is beyond the scope of the original metric. ${ }^{12}$ The number of followers for the individuals captured in the original $\mathrm{K}$-index paper is much lower than those of the journals included in these analyses. Therefore, the K-index scores may have been unrepresentatively high. The Grey Scale is a rudimentary metric for the examination of impact factor and Twitter followers based on the available general medical journals; therefore, it may not be generalizable to other journal types.

There were a number of outlying journals that showed disproportionately high numbers of fol-

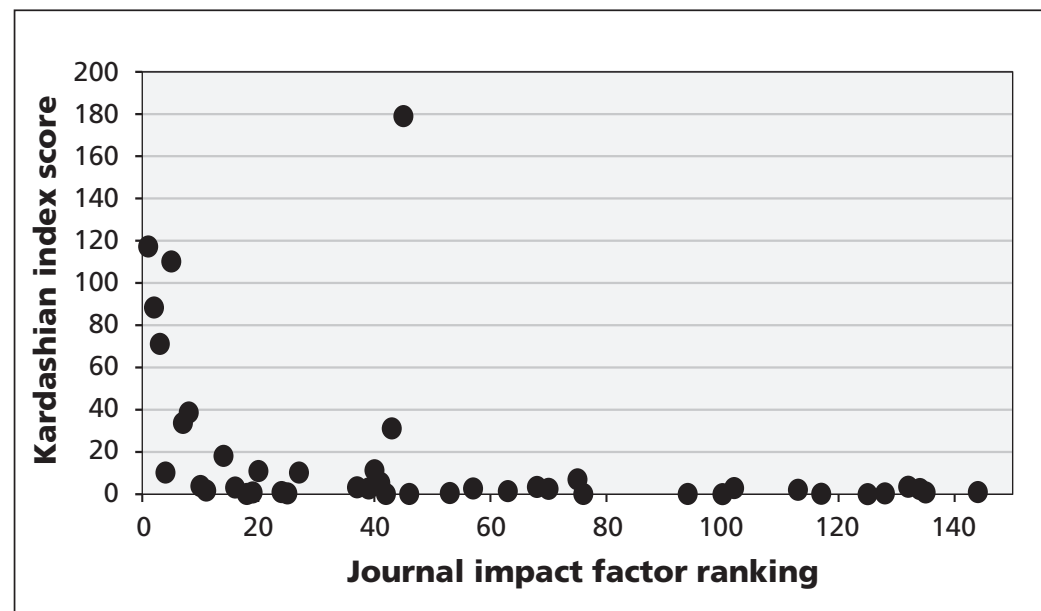

Figure 3: Scatterplot of Kardashian Index score and journal impact factor ranking.

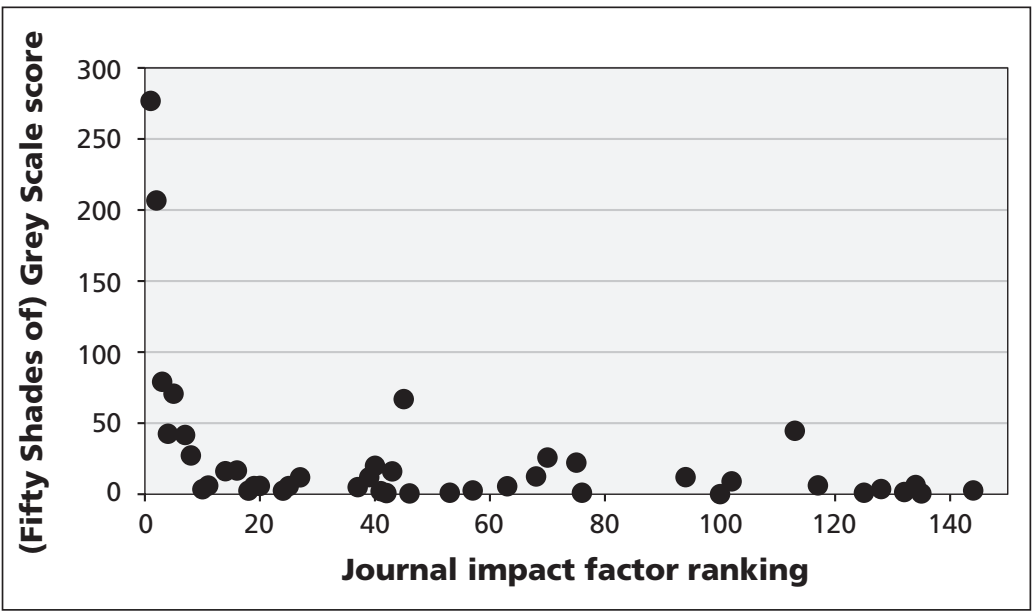

Figure 4: Scatterplot of the (Fifty Shades of) Grey Scale score and journal impact factor ranking. lowers despite their comparatively low impact factor and citations - Hall's so-called Science Kardashians. ${ }^{12}$ This moniker may be somewhat harsh; despite their discrepant mainstream popularity, these journals are generally regarded as reputable. For this reason, the K-index threshold of 5 or more may be misleading. The present study provides an alternative and data-driven means with which to examine outliers, such as by using a $99 \%$ confidence interval. Further refinement of the K-index and the Grey Scale is warranted. Cognisant that these metrics have been proposed in jest, the K-index and Grey Scale do, however, prod at the tender underbelly of science's unspoken popularity contest.

The demonstration of a positive relation between mainstream popularity and scientific merit is encouraging. It must, however, be acknowledged that the measures used to capture this relation - Twitter followers and journal impact and citations - use proxies. Although social media use is incredibly pervasive, less than a third of general medical journals have used Twitter, so this may not be the best proxy for mainstream media popularity. There has been some controversy over the use (and abuse) of impact factor as a meaningful metric for capturing scientific merit, ${ }^{17,18}$ suggesting that further refinement is warranted. High-quality journals are garnering the greatest online followings, which hopefully will translate into a greater number of people absorbing high-quality, evidence-based research. These results stand in contrast to a recent study of urology journal impact factors and Twitter followings, where no relation was seen. ${ }^{16}$ That study included just 8 journals compared with 43 in the current study, which may have resulted in reduced statistical power concealing the relation. ${ }^{16}$ Further investigation into the mechanisms and direction of causality in this relation is warranted.

The original application of the K-index was to individuals, identifying discrepant social media popularities. ${ }^{12}$ Many people captured in the Hall study have media profiles that expand beyond their scientific work; for example Neil deGrasse Tyson(@neiltyson) has, among other public appearances, hosted the science-based radio program "StarTalk." In the current study, these methods have been applied to medical journals, eliminating variables beyond scientific merit, such as humour, which people often show in their Twitter posts. A metric specific to the examination of journals has been developed in the current study, highlighting the association between a journal's scientific merit and its mainstream popularity.

With only $28.1 \%$ of general medical journals hosting a Twitter profile, this means of dissemina- 
tion is greatly underused. These results are in line with the Twitter participation of urological journals $(24.2 \%) .{ }^{16}$ However, the uptake of Twitter among physicians is on the rise, ${ }^{19}$ a trend that will hopefully extend to medical journals. Rather than identifying large numbers of Science Kardashians, the present study shows that many more journals are closer to "Popularity Franklins" - they have received disproportionately low levels of recognition and popularity than would be warranted by their scientific merit, as per Rosalind Franklin. One of the considerable barriers between medical research and the general public is the means of communication and knowledge translation. Twitter is a hospitable middle ground where the lay reader need not pore through a peerreviewed journal to extract its actionable pieces of information, 140 characters at a time..$^{9,19}$

\section{Conclusion}

There is a positive relation between the scientific merit of general medical journals and the journal's Twitter following. Twitter is an underused means of communication, with less than a third of medical journals hosting a profile. With the exception of a few outliers, most journals had Twitter followings that corresponded with their impact factor and citations. These results suggest that higher impact science is reaching a greater proportion of the general public than lower impact science. In an era in which engaging in social media has become a part of medical research, the demonstration of Twitter as an effective means of research dissemination may make Beliebers of us all.

\section{References}

1. Launer J. The age of Twitter. Postgrad Med J 2013;89:675-6.

2. Lulic I, Kovic I. Analysis of emergency physicians' Twitter accounts. Emerg Med J 2013;30:371-6.

3. St Louis C, Zorlu G. Can Twitter predict disease outbreaks? BMJ 2012;344:e2353.
4. Finfgeld-Connett D. Twitter and health science research. West $J$ Nurs Res 2015;37:1269-83.

5. Gomes C, Coustasse A. Tweeting and treating: how hospitals use twitter to improve care. Health Care Manag (Frederick) 2015;34:203-14.

6. Forgie SE, Duff JP, Ross S. Twelve tips for using Twitter as a learning tool in medical education. Med Teach 2013;35:8-14.

7. King D, Ramirez-Cano D, Greaves F, et al. Twitter and the health reforms in the English National Health Service. Health Policy 2013;110:291-7.

8. Twitter [homepage]. Available: https://about.twitter.com/company. (accessed 2015 July 27).

9. Bosley JC, Zhao NW, Hill S, et al. Decoding twitter: surveillance and trends for cardiac arrest and resuscitation communication. Resuscitation 2013;84:206-12.

10. Ranney ML, Daya M. Twitter and resuscitation education: Is this the future? Resuscitation 2013;84:147-8.

11. Justin Bieber discography. Wikipedia [updated 2015 Oct. 17]. Available: https://en.wikipedia.org/wiki/Justin_Bieber_discography (accessed 2015 Oct. 10).

12. Hall N. The Kardashian index: a measure of discrepant social media profile for scientists. Genome Biol 2014;15:424.

13. Flood A. Fifty Shades of Grey sequel breaks sales records. London: The Guardian; 2015 June 23. Available: www.theguardian .com/books/2015/jun/23/fifty-shades-of-grey-sequel-breaks-sales -records (accessed 2015 Aug. 5).

14. Garfield E. Citation analysis as a tool in journal evaluation. Science 1972;178:471-9.

15. Eysenbach G. Can tweets predict citations? Metrics of social impact based on Twitter and correlation with traditional metrics of scientific impact. J Med Internet Res 2011;13:e123.

16. Nason GJ, O'Kelly F, Kelly ME, et al. The emerging use of Twitter by urological journals. BJU Int 2015;115:486-90.

17. Elliott DB. The impact factor: a useful indicator of journal quality or fatally flawed? Ophthalmic Physiol Opt 2014;34:4-7.

18. Baethge C. Impact factor - a useful tool, but not for all purposes. Dtsch Arztebl Int 2012;109:267-9.

19. Choo EK, Ranney ML, Chan TM, et al. Twitter as a tool for communication and knowledge exchange in academic medicine: a guide for skeptics and novices. Med Teach 2015;37:411-6.

Affiliations: Telecommunications, Web \& Internet Trust; and the Medical Research Council Unit for Lifelong Health \& Ageing, University College London, London, UK

Ethical approval: No Twitterati were harmed in the production of this manuscript. All data are freely available online.

Acknowledgements: The author thanks Madeleine Cosco, for her invaluable input in the coining of the (Fifty Shades of) Grey Scale and her tolerance of innumerable Kardashianand Shades-related discussions; his fellow Lifelong Health \& Aging postdocs, for producing the muted chuckle that catalyzed the production of this paper; and Sainsbury's Pink Bolts, for the caffeinated calorie-less splendour that enabled the preparation of this manuscript. 